\title{
ERRATA
}

\section{Erratum: Radiation emitted by quantum-well InGaAs structures \\ I. Spontaneous emission spectra [Semiconductors 32, 423-427 (April 1998)]}

P. G. Eliseev

Temporary Address: Center of High-Technology Materials, University of New Mexico, Albuquerque, New Mexico, USA

\section{V. Akimova}

P. N. Lebedev Physics Institute, Russian Academy of Sciences, 117924 Moscow, Russia

Fiz. Tekh. Poluprovodn. 32, 1408 (November 1998)

[S1063-7826(98)02411-9]

The correct figures for this article should read as follows:

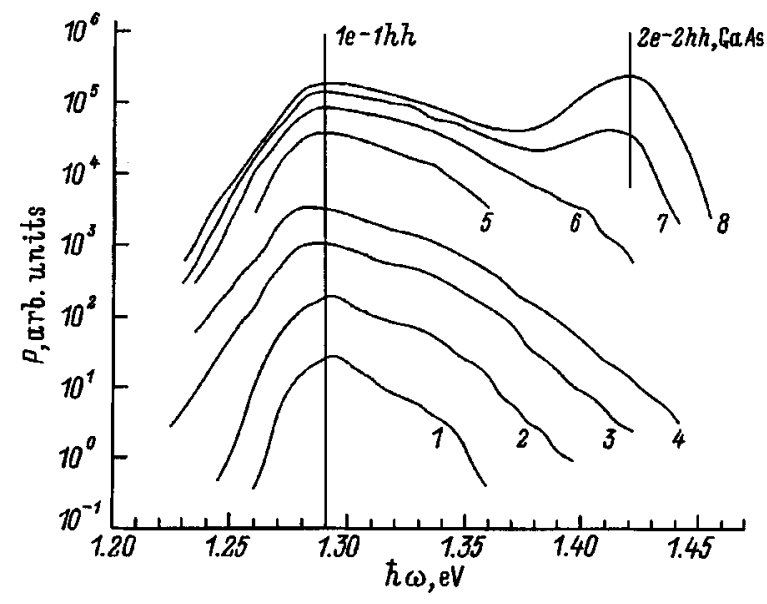

FIG. 1. Relative spectral density $P$ of the radiation of an InGaAs quantum well at $286 \mathrm{~K}$. Pump current $I$, mA: $1-1,2-2,3-5,4-10,5-50$, $6-100,7-150,8-200$. The spectra 3 and 4 were obtained in the direct-current regime.

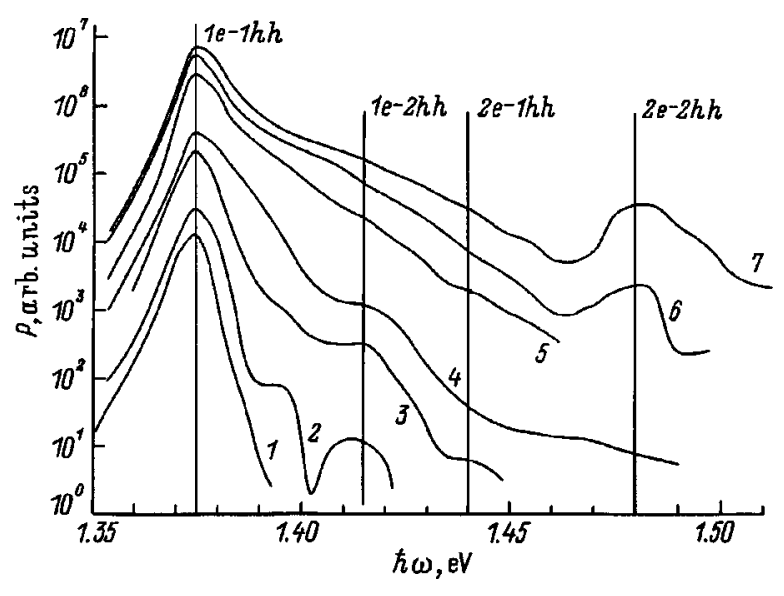

FIG. 2. Relative spectral density $P$ of the radiation of an InGaAs quantum well at $4.2 \mathrm{~K}$. Pump current $I$, mA: $1-1,2-2,3-10,4-20,5-$ $100,6-150,7-200$. The spectra 1 and 4 were obtained in the direct current regime. 